\title{
Wavelet analysis of stellar differential rotation
}

\section{The Sun in white light}

\author{
A. Hempelmann ${ }^{\star}$ \\ Universität Hamburg, Hamburger Sternwarte, Gojenbergsweg 112, 21029 Hamburg, Germany \\ Received 20 September 2002 / Accepted 27 November 2002

\begin{abstract}
Future space projects like KEPLER will deliver a vast quantity of high precision light curves of stars. This paper describes a test concerning the observability of rotation and even differential rotation of slowly rotating stars from such data. Two published light curves of solar total irradiance measures are investigated: the Nimbus-7 Earth Radiation Budget (ERB) observations between 1978 and 1993 and the Active Cavity Radiometer Irradiance Monitor I (ACRIM I) measurements between 1980 and 1989. Light curve analysis show that oscillations on time-scales comparable to solar rotation but of a complex pattern are visible. Neither Fourier analysis nor time-frequency Wavelet analysis yield the true rotation period during the more active phases of the solar cycle. The true rotation period dominates only for a short time during solar minimum. In the light of this study even space-born broad band photometry may turn out an inappropriate instrument to study stellar butterfly diagrams of stars rotating as slow as the Sun. However, it was shown in Papers I and II of this series that chromospheric tracers like Lyman $\alpha$, $\mathrm{Mg}$ II $\mathrm{h}+\mathrm{k}$ and $\mathrm{CaII} \mathrm{H}+\mathrm{K}$ are appropriate instruments to perform this task.
\end{abstract}

Key words. Sun: rotation - stars: rotation - methods: data analysis

\section{Introduction}

Stellar activity as an analogue to solar activity is observed through wide ranges of the HRD. X-ray observations such as the ROSAT All Sky Survey have shown that all cool stars of the solar neighborhood exhibit a hot corona (Schmitt 1997). An X-ray emitting corona is considered to be the most serious indicator of magnetically induced activity: all attempts to explain the phenomenon of coronal heating without magnetic fields have failed. Stellar rotation is the most important factor controlling the level of X-ray luminosity (and other tracers of stellar activity); introduction of the convective turnover time as the second basic parameter of the stellar dynamo gives only a marginally improved relationship (Hempelmann et al. 1995). Doppler tomography as well as broad-band photometry have shown that rapidly rotating stars are typically covered by one or more large extended spots often located near the rotation pole (cf. Strassmeier 1996). These spots cause a modulation of the stellar brightness easily detectable by broad-band photometry, enabling determination of the rotation period. Monitoring the period over at least one activity cycle can yield a stellar butterfly diagram. A butterfly diagram is a combination of two effects: stellar surface differential rotation and systematic latitudinal shift of active belts over an activity cycle. Automatic photometric terrestrial telescopes are an efficient tool for the observation of butterfly diagrams (cf., for example, Strassmeier et al. 1997).
Unfortunately, the photometric method can only be applied to rapidly rotating, i.e., active stars. Low-activity stars like the Sun show amplitudes of variability (Radick et al. 1990) which cannot be detected by terrestrial telescopes with typical detection limit of one mmag. This causes a blind spot on the map in the range of slow rotators concerning activity and differential rotation. Almost all knowledge of activity and differential rotation of these stars is based on CaII $\mathrm{H}+\mathrm{K}$ observations (Baliunas et al. 1995; Baliunas et al. 1985; Donahue et al. 1996). On the other hand, the Sun is a slow rotator and if we want to know what to expect from the Sun in the future we have to study stars rotating as slow as the Sun.

The question whether photometry is an appropriate tool to detect stellar rotation or even differential rotation of slowly rotating stars must be asked anew in the light of future space missions. Several missions planned by NASA and ESA shall monitor (as by-product) up to millions of stars over years with a photometric accuracy up to $0.01 \mathrm{mmag}$. An example is the KEPLER mission where about $100000 \mathrm{G}$ dwarfs shall be observed over years with a precision of $10 \mu \mathrm{mag}$ (D. Soderblom, private communication). Hence one can guess that this precision is sufficient to observe rotational modulation also for stars rotating as slow as the Sun. The question, however, is whether these stars indeed produce rotational modulation or not and, whether this modulation mirrors veridical the rotation period or even the pattern of differential rotation on the stellar surface.

\footnotetext{
^ e-mail: ahempelmann@hs.uni-hamburg.de
} 


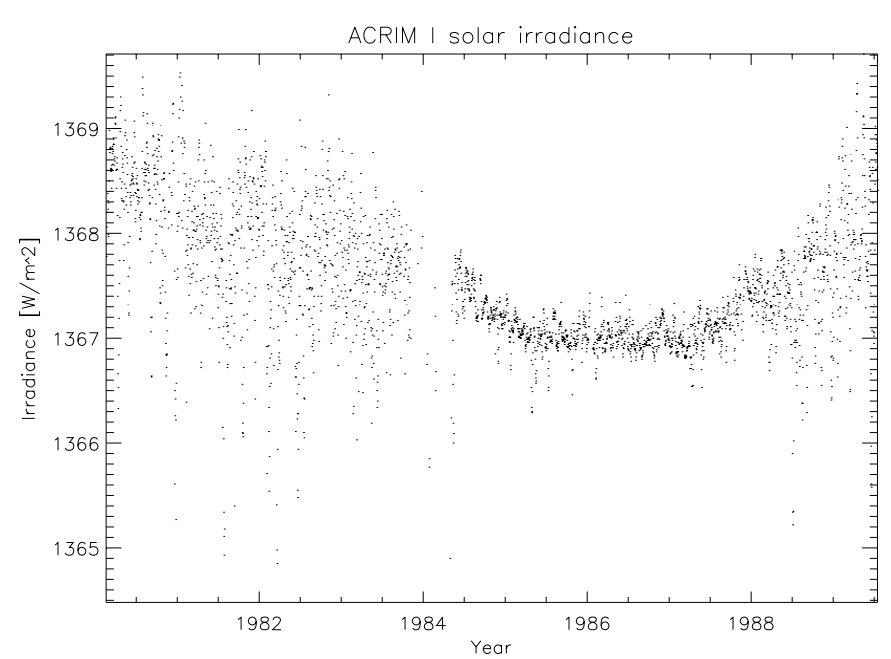

Fig. 1. Time-series of ACRIM I solar irradiance measures.

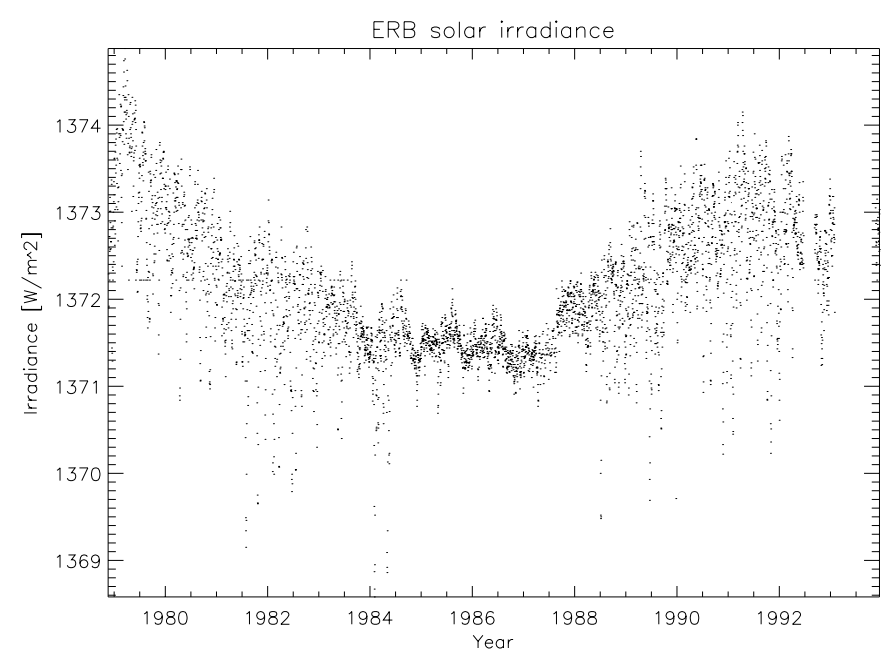

Fig. 2. Time-series of ERB solar irradiance measures.

To test this I analyse white light observations of the Sun which have an accuracy comparable to the planned and above mentioned space missions. These are monitoring records of the total solar irradiance.

\section{Observations}

Four data records are published and available via the Internet: http://daac.gsfc.nasa.gov/CAMPAIGNDOCS/FTPSITE/ INTDIS/readmes/solirrad.html. These are the Active Cavity Radiometer Irradiance Monitors (ACRIM) I and II on board of the Solar Maximum Mission (SMM) spacecraft respectively the Upper Atmosphere Research Satellite (UARS). There are two further datasets independent of the ACRIM observations: from the Nimbus-7 Earth radiation Budget (ERB) instrument and from the Earth Radiation Budget Satellite (ERBS). In this paper the ACRIM I and the ERB observations are analysed. The reasons for this choice are: i) they are independent measures, ii) the records are long and dense, iii) the ACRIM I data span a time interval between 1980 and 1989 which is completely enclosed in the ERB record (1979-1992), iv) fluctuations on time-scales comparable with the solar

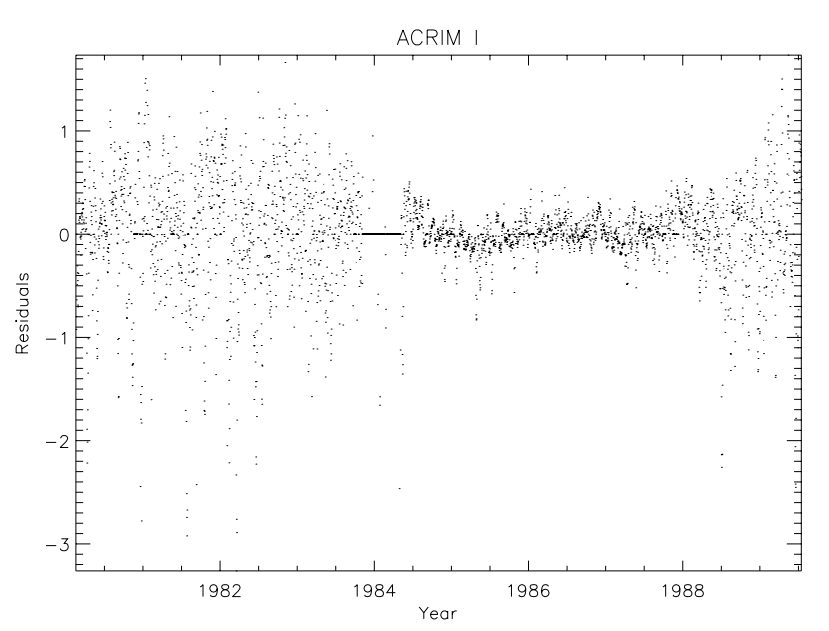

Fig. 3. The ACRIM I data after removal of long-term variability.

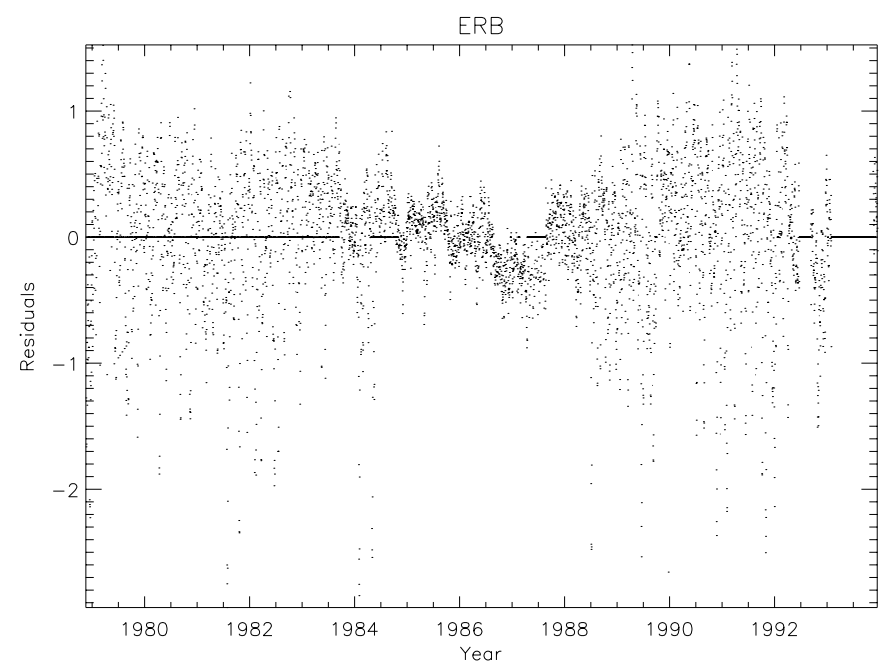

Fig. 4. The ERB data after removal of long-term variability.

rotation are clearly visible which suggests that they really are caused by rotation and, v) the ACRIM observations are self-calibrated whereas the ERB and ERBS show degradation not removed from the data and, hence the question can be answered whether such additional modulation will hamper an investigation.

The ACRIM I experiment consists of three independent, electrically self-calibrated cavity pyrheliometers (Willson 1981) with a cavity absorptance of 0.9995 from the UV through the far IR (Zelewski et al. 1979). The individual sensors have been described by Willson 1979 and Willson 1980. Data basis are daily means of $10^{4}$ individual measures with an rms error of $1-2 \times 10^{-5}$ (Willson \& Hudson 1991).

The ERB instrument is different in design and operation. The main characteristics are that the instrument is not selfcalibrating and not pointed to the Sun. Calibration is based on two sources: a deep space reference and an artificial source of heating. Observation is a scanning procedure. During each orbit the Sun moves through the field of view for a few minutes, thus the signal varies with the cosine of the Sun's off-axis angle. The averaged signal per orbit serves as data basis of the 


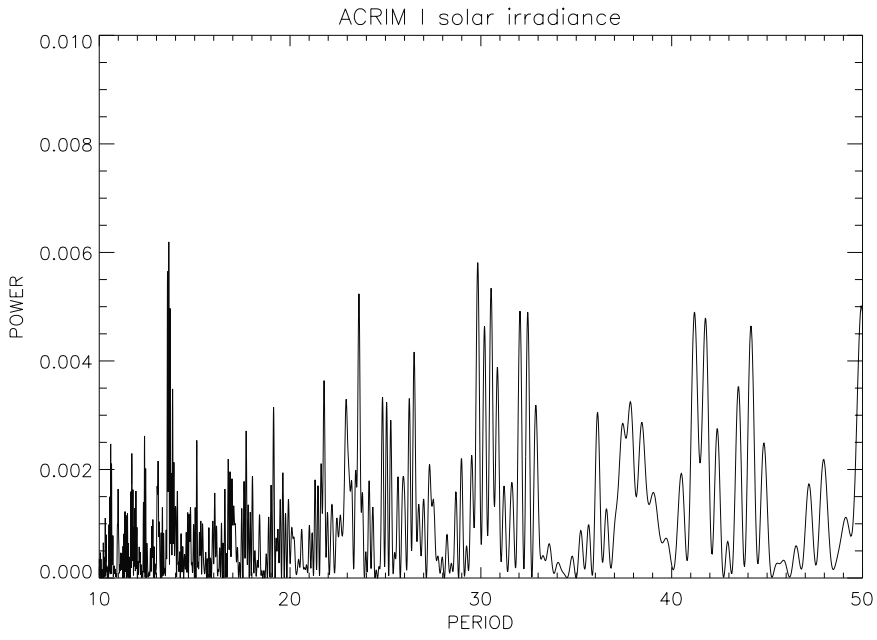

Fig. 5. The ACRIM I Fourier spectrum.

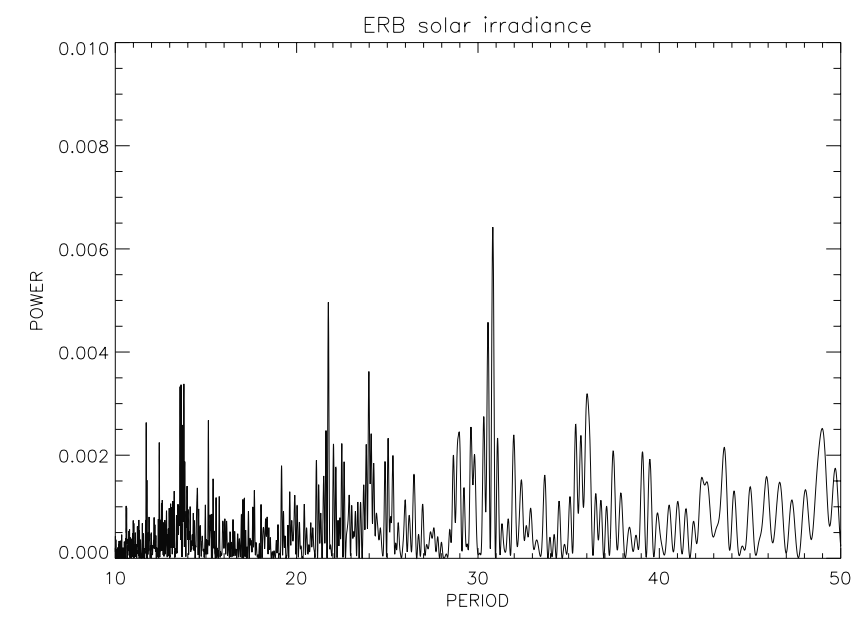

Fig. 6. The ERB Fourier spectrum.

published daily means (Hoyt et al. 1992). The accuracy of the data is of the order of $10^{-4}$ (Hoyt et al. 1992). While these data are less precise in comparison to ACRIM I, the ERB timeseries is longer and includes a longer interval of increasing solar activity during solar cycle 22.

The phenomena of ERB sensor degradations were discussed by several authors, (cf., e.g. Willson \& Hudson 1991; Willson 1994; Willson 1997; Lee III et al. 1995; Mecherikunnel 1994). While the effects on the ACRIM data are removed by self-calibrations (Willson 1994) they remain as more or less linear trends in the ERB data holding for several solar rotations. This may affect period analysis and it will be interesting to check its influence from a comparison with the ACRIM I result.

Figures 1 and 2 show the two time-series to be analysed. Long-term variations reflecting the solar cycle were removed by fitting low-order polynomials and setting the mean values to zero and filling in the gaps with zero (Figs. 3 and 4). These figures clearly show irradiance variability on short time-scales which was interpreted as rotational modulation and active region life times (Willson 1997). Note that the amplitude of

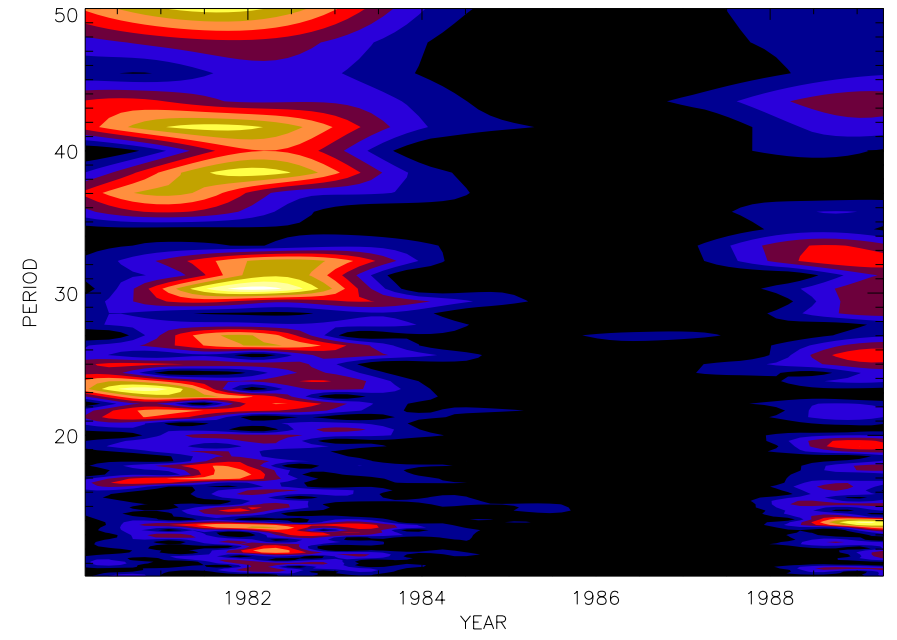

Fig. 7. Wavelet map of the ACRIM I solar irradiance data.

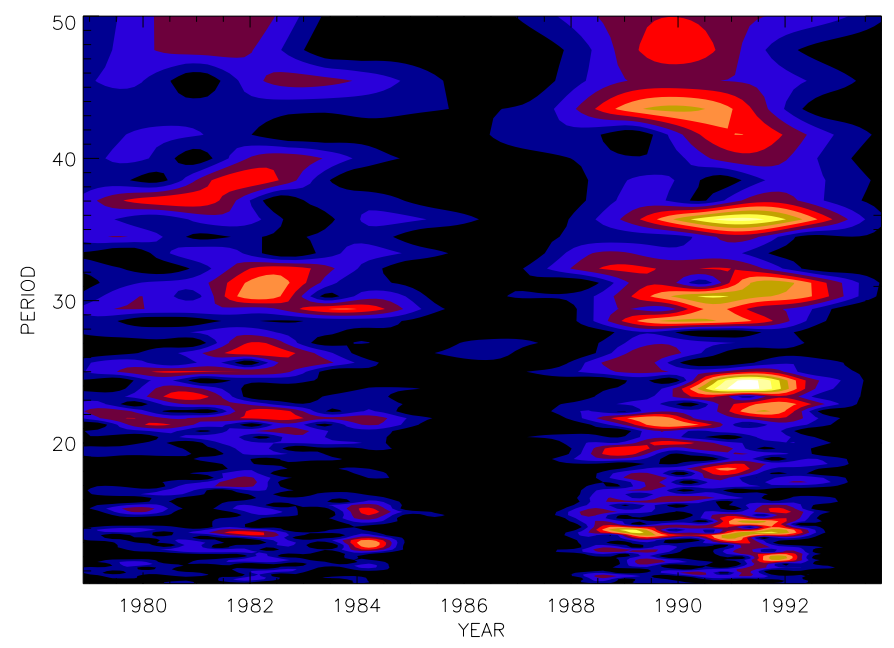

Fig. 8. Wavelet map of the ERB solar irradiance data.

the short-term fluctuations strongly depends on the phase of the solar cycle.

\section{Time-frequency analysis}

As a first step a Fourier analysis was performed to see whether the known solar rotation period is evident in the data (Figs. 5 and 6). Both power spectra show a number of peaks. In the ACRIM I spectrum no obviously dominant single peak is visible. Maximum power is at $\mathbf{1 3 . 6 5}$ days, exactly half of what is expected. Next peak is 29.8 days which is $2-3$ days larger as the solar synodic rotation. There are further peaks in the range 23-45 days which are almost as strong as the 30 days peak. The ERB spectrum shows a dominant peak at 30.8 days, i.e., also only in the vicinity of the true rotation period. A second clear peak is at 21.7 days. Half the rotation period is also visible.

A Wavelet analysis was carried out in the same manner as described in Papers I and II (Hempelmann \& Donahue 1997; Hempelmann 2002) and with the same time and frequency (period) resolution of 300 resp. 0.5 days. Figures 7 and 8 show the wavelet maps. 
At first glance the structure of the two maps is very similar: during solar minimum there is almost no oscillation visible while during solar maximum the wavelet maps show ridges of enhanced power indicating oscillations. These oscillations appear in both maps but with differing intensities. This difference is due to different data structures. The ACRIM I data are more precise and also more dense over solar cycle 21 . Hence the ACRIM I map shows stronger contrast in this region. However, during cycle 22, the ERB data are as dense as the ACRIM I data but the ERB time series is much longer. Therefore the ERB wavelet map exhibits a region of good contrast after 1989 where the ACRIM I observations had already been finished.

The pattern of oscillations is the same as that exhibited in the Fourier spectra: there is a plethora of peaks (ridges) where no single period dominates over the whole time span. The true rotation period of 27 days is visible but it is at almost all times of minor power in comparison with oscillations of shorter or longer periods. The period values of the oscillations are (ERB values in brackets): 12 (12), 13.5 (13.5), 17-18 (15), 23 (22), 27 (26), 30 (30), 38 (38), 41 (41) and 50 (50) days during solar cycle 21 . During cycle 22 one can find (ACRIM I values in brackets): 12, 13.5-14.5 (13.5), 18, 24 (25), 29 (29), 30 (30), 36 (36), 43 (41-43) and 49 days.

\section{Discussion}

First of all one has to note that the results do not differ basically between ACRIM I and ERB nor between cycles 21 and 22. Hence the reduced precision of ERB data where sensor degradation effects were not removed has no significant influence on these results. This similar structure is partly already apparent in the Fourier analysis: all peaks of enhanced power in the diagram of the less accurate ERB data appear in the ACRIM I diagram, too. The latter diagram showing further peaks may be the result of higher accuracy of the ACRIM I observations which could exhibit more details of the oscillations pattern inherent in the data. This qualitative agreement is less clear concerning the amplitudes: the ACRIM I spectrum has its maximum at 13.7 days (exactly half the rotation period) while the ERB spektrum has its maximum at a period of 30.9 days which, in the ACRIM I spectrum, is the second powerful peak of almost the same height as the 13.7 days period. However, the 13.7 peak is also visible in the ERB spectrum but split into two peaks of 13.6 and 13.8 days.

The true solar rotation period is visible as a minor power peak in the ACRIM I Fourier spectrum but not visible in the ERB Fourier spectrum. This is not the case in the Wavelet map. Both the ACRIM I map and the ERB map show a 27 days ridge around 1982 and 1986-87. While in 1982 other periods dominate it is the dominating period during 1986-87 at solar minimum where both maps are empty at all other periods. If the time resolution of one and a half year is taken into account, the true solar rotation was detectected only as a snapshot during a very short part of the 11 years cycle.

The source of the dominating oscillations different from the true rotation period is likely to be intrinsic because of the very similar spectra of the two independent data sets. Two sources are suspected: i) rapid evolution of dark spots on time scales which are comparable with the rotation period and, ii) correlated contributions of dark spots and bright faculae to the light curve.

It was shown in Paper II of this series (Hempelmann 2002) that a phase shift causes a shift of the observed period; the more rapid this shift, the bigger the difference between the observed period and the true one. At solar maximum when the Sun is covered by a large number of spots or spot groups their intrinsic evolution will cause a steady oscillation of the centroid of spot patterns which will be reflected in a corresponding oscillation of the phase of rotational modulation. Faculae, however, are stable over longer times.

The structure of an active region consisting of larger extended areas of faculae and more concentrated regions of spots may also influence the corresponding light curve to an extent where deviations from a simple sine curve with 27 days period will be visible. As noted by Willson (1997) the rotational modulation of the total solar irradiance is caused by dark spots as well as bright faculae. When an active region appears at the solar limb, brightening is observed caused by preceding faculae. This increasing brightening will be followed by a darkening from spots when they appear at the solar limb. When these spots disappear at the opposite limb a second brightening will be observed because the net balance is again determined by faculae alone. Finally the brightening will disappear when the faculae disappear at the solar limb. If this scenario is correct the whole light curve would tend to form a double wave rather than a single wave caused by a single dark spot (or spot group) on a rotating star. This may explain the strong appearance of half the rotation period in the data. It seems that only in cases where either dark spots totally dominate (young and active stars) or faculae do (weakly active stars during activity minimum) the right rotation period dominates rotational modulation and can therefore be observed from light curves.

While the contributions of dark and bright sources are merged in the light curve of a rotating star, rotational variability of a chromospheric tracer is caused solely by bright sources. This is probably one reason why the true rotation period of the Sun and even differential rotation could be detected from timeseries of Ca II K (Paper I) as well as Lyman $\alpha$ and Mg II h+k (Paper II).

\section{Conclusions}

Photometry is a powerful tool to find the rotation period or even differential rotation of an active star, where extended dark spots dominate the light curve and cause observable amplitudes which are stable over many stellar rotations. From this analysis of solar irradiance data, however, it seems doubtful whether photometry can be succesfully applied in the same manner to stars with solar-like activity levels. This is not a question of data acquisition accuracy but of stellar intrinsic nature. The reasons may be a variable net balance of dark spots and bright faculae as well as a rapid evolution of spots on time-scales comparable to stellar rotation. Thus it may be doubted whether future space missions delivering a vast quantity of stellar light curves will noticeably contribute to rotation periods of slowly rotating, i.e. weakly active stars. As shown in Papers I and II, observation 
of chromospheric activity tracers resulting mainly from plage seem to be the more promising method. This strongly suggests the use of spectroscopy rather than broad-band photometry for studying the rotation of low-active stars.

Acknowledgements. The author wish to thank John R. Hickey and the Nimbus-7 ERB Experiment Team for their solar data; Richard C. Willson for the ACRIM I solar data, and the Distributed Active Archive Center (code 902.2) at the Goddard Space Flight Center, Greenbelt, MD, 20771, USA, for putting these data in their present format and distributing them. The production and distribution of these data were sponsored by NASA's Earth Science enterprise.

\section{References}

Baliunas, S. L., Horne, J. H., Porter, A., et al. 1985, ApJ, 294, 310

Baliunas, S. L., Donahue, R. A., Soon, W. H., et al. 1995, ApJ, 438, 269

Donahue, R. A., Saar, S. H., \& Baliunas, S. L. 1996, ApJ, 466, 384

Hempelmann, A., Schmitt, J. H. M. M., Schultz, M., Rüdiger, G., \& Stępień, K. 1995, A\&A, 294, 515
Hempelmann, A., \& Donahue, R. A. 1997, A\&A, 322, 835 (Paper I) Hempelmann, A. 2002, A\&A, 388, 540 (Paper II)

Lee III, R. B., Gibson, M. A., Wilson, R. S., \& Thomas, S. 1995, J. Geophys. Res., 100, 1667

Mecherikunnel, A. T. 1994, Sol. Phys., 155, 211

Radick, R. R., Lockwood, G. W., \& Baliunas, S. L. 1990, Science, 24, 39

Schmitt, J. H. M. M. 1997, A\&A, 318, 215

Strassmeier, K. G. 1996, Stellar Surface Structure, ed. K. G. Strassmeier, \& J. L. Linsky, Proc. IAU Coll., 176, 289

Strassmeier, K. G., Bartus, J., Cutispoto, G., \& Rodono, M. 1997, A\&AS, 125, 11

Willson, R. C. 1979, Applied Optics, 18, 179

Willson, R. C. 1980, Applied Optics, 19, 3256

Willson, R. C. 1981, Sol. Phys., 74, 217

Willson, R. C., \& Hudson, H. S. 1991, Nature, 351, 42

Willson, R. C. 1994, Proc. IAU Coll., 143, 54

Willson, R. C. 1997, Science, 277, 1963

Zelewski, E., Geist, J., \& Willson, R. C. 1979, Proc. Soc. Phot. Opt. Instr., 196, 152 